\title{
Infection in prosthetic material
}

George Smith MD MRCS is an NIHR Academic Clinical Lecturer in the Academic Vascular Surgery Unit, Hull and East Yorkshire NHS Trust. Conflicts of interest: none.

Ian Chetter MD FRCS is Professor of Surgery at University of Hull / Hull York Medical School and Honorary Consultant Vascular Surgeon in the Academic Vascular Surgery Unit, Hull and East Yorkshire NHS Trust. He is also Training Programme Director for vascular surgery in Yorkshire and the Humber deanery and member of the education committee of the Vascular Society of Great Britain and Ireland. Conflicts of interest: none.

We are grateful to Miss Rachel Barnes for her work on the previous article of the same title.

\section{Abstract}

Surgical site infection (SSI) occurs when a wound created as part of a surgical procedure becomes infected. SSI is one of the most common healthcare associated infections and occurs in approximately $5 \%$ of patients undergoing a surgical procedure. SSI may lead to patients suffering considerable morbidity or mortality and have significant cost implications. The aetiology involves the interplay of host, environmental and pathogen factors all of which should be addressed in seeking to reduce the risk of developing an infection. The presence of prosthetic material reduces the number of bacteria necessary for an infection to develop and can give rise to treatment and diagnostic difficulties. The responsible organisms are most commonly Staph aureus and Staph epidermidis. Diagnosis is frequently problematic and antibiotic treatment alone is often ineffective due to biofilm formation necessitating removal of prosthesis in many cases. Prevention of infection is by far the most important aspect of prosthetic implant surgery. Patient optimisation is equally important as the cutting edge research into biological prostheses in reducing the incidence of prosthetic infection in future practice.

\section{Keywords}

Prosthesis; infection; surgery; radiology; vascular, orthopaedics

\section{Introduction}

The Centre for Disease Control defines a Surgical Site Infection (SSI) as an infection within 30 days of an operation or up to one year if a surgical prosthesis is implanted during the procedure. Surgical site infections reportedly occur in $5 \%$ of patients leading to considerable morbidity and over a third of post-operative deaths are related, at least in part, to an SSI [1]. However it has been suggested that when close post discharge surveillance is undertaken, 
SSI complicate $10-20 \%$ of surgical procedures indicating an underestimation of the incidence within all surgical specialties[2].

A surgical prosthesis is defined as 'artificial material which is used to replace missing part or parts after surgical intervention' and includes vascular grafts, orthopaedic appliances and synthetic mesh. Careful consideration must be given to the need for insertion of prosthetic material as it acts as a foreign body within the surgical wound and studies have previously demonstrated a logarithmic decrease in the number of bacteria required to cause an SSI in the presence of a prosthesis [3].

The actual risk of developing a SSI in the presence of a surgical prosthesis is unknown, but approximately half of healthcare associated infections in the US are reportedly related to surgery involving prosthetic material. Current literature quotes an SSI incidence of between 0.2 and $6 \%$ in vascular grafts [4], 0.3-1.9\% in knee and hip arthroplasties[5] and approximately $1.25 \%$ in cardiac device[6].

\section{Factors influencing prosthetic infection}

Many factors influence the healing of surgical wounds and hence the potential for and incidence of SSI and prosthetic implant infection. Patient factors known to increase the incidence of SSI are summarised in table 1.

Table 1: Risk factors for the development of surgical site infections

\begin{tabular}{|l|l|}
\hline Patient Risk Factors & \\
\hline Age & Peak at 65 yrs due to $\uparrow$ co-morbidities \\
\hline Smoking & Platelet aggregation and $\downarrow$ oxygen carriage \\
\hline Nutrition & $\begin{array}{l}\text { Hypoalbuminaemia }<30 \mathrm{mg} / \mathrm{dl} \text { is independent risk factor } \\
\text { for SSI }\end{array}$ \\
\hline Obesity & $\downarrow$ tissue oxygen tension levels \\
\hline Diabetes & $\begin{array}{l}\downarrow \text { tissue perfusion and impaired cellular immune } \\
\text { response }\end{array}$ \\
\hline Corticosteroids & $\begin{array}{l}\downarrow \text { vascular permeability and impaired cellular immune } \\
\text { response }\end{array}$ \\
\hline Burns/trauma & Impaired cellular immune response \\
\hline Neoplasia/ chemo-radiotherapy & Impaired cellular immune response \\
\hline
\end{tabular}

\section{Risk stratification}

The National Academy of Sciences classification allocates all surgical wounds to one of 4 categories - clean, clean contaminated, contaminated and dirty. SSI incidence varies from $<2 \%$ for clean wounds compared to up to $40 \%$ for dirty wounds.

The National Nosocomial Infections Surveillance System risk index for the development of SSI comprises a 0-3 score using the following three risk factors.

a) An pre-operative assessment score of 3, 4 or 5 in the five category American Society of Anesthesiologists' system 
b) Wound classified as contaminated or dirty

c) Procedure lasting more than a specific period of time ('T hours'), where $T$ is the 75th percentile of the duration of the surgery being performed surgery.

\section{Pathogenesis}

When a prosthesis is implanted it is immediately surrounded by a pool of blood and inflammatory fluid. Initial fibrinogen adherence facilitates formation of a proteinaceous layer consisting of platelets and red blood cells. Subsequent white blood cell adherence to this layer leads to bacterial adhesion and the possibility of prosthetic infection[7]. Different prostheses display differing critical surface tensions. A higher surface tension results in greater adherence of fibrinogen and platelets and the subsequent risk of bacterial colonisation and prosthetic infection. Coagulase negative staphylococci, are the most common causative pathogens responsible for 'clean' implant surgery. This is because they possess surface proteins which have adhesive properties and facilitate the initial colonisation of prostheses [8].

Non-haemolytic S. epidermidis has been determined to be the single most common pathogen in the development of prosthesis material infections[9]. This is due to the ability to produce an exopolysaccharide (slime) which coats the prosthesis and in doing so facilitates further bacterial adhesion leading to formation of a biofilm[10]. This biofilm has been shown to promote the adherence of other bacteria to a prosthesis and also to render the micro-organisms less susceptible to circulating antimicrobials. The biofilm decreases chemotaxis and opsonization of neutrophil granulocytes, increases degranulation, and blocks antibiotic penetration into the bacterial cell[10]. The nature of the biofilm can make isolation of the bacteria on blood culture difficult and strains are often only isolated after removal and culture of the prosthetic material. This hampers diagnosis and is compounded by the long latent period and indolent nature of S. epidermidis infection.

Staphylococcus aureus (S. aureus) is a gram-positive, coagulase positive coccus. Many isolates exist, each have their own virulence factors and hence each responsible for the development of differing infections. Along with factors allowing adherence to epithelial and endothelial lined surfaces, most strains also possess a fibronectin and fibrinogen binding protein promoting adherence to blood clots and traumatised tissues. S. aureus accounts for $30 \%$ of all soft tissue SSI and thus is a frequent isolate in prosthetic infections [11]. Methicillin Resistant S. aureus (MRSA) is a strain of S. aureus which has developed resistance to the actions of methicillin and other $\beta$-lactam antibiotics. Almost half $(49.2 \%)$ of prosthetic infections due to $s$. aureus are due to MRSA strains [11]. Some $S$. aureus strains have even developed resistance to vancomycin and are termed VRSA (Vancomycin resistant S. aureus).

Organisms such Streptococci, diphtheroids and enterococci each account for approximately $10 \%$ of ?prosthetic infections, whilst gram-negative cases are much less common. Anaerobes are usually only found as components of a polymicrobial infection. 


\section{Prevention}

Interest in SSI and prosthetic infection has increased in recent years and a growing evidence base is available for interventions which may impact upon the incidence of infection following surgery. However, seemingly obvious questions such as the best methods for reducing bacterial load in the area of the incision or timing of administering antibiotics are still to be conclusively answered. Prevention strategies are summarised in Table 2 .

Table 2. Strategies to prevent surgical site infections

\begin{tabular}{|c|c|}
\hline \multicolumn{2}{|l|}{ Pre operative } \\
\hline $\begin{array}{l}\text { MRSA screening and } \\
\text { decontamination }\end{array}$ & Shown to reduce incidence of MRSA SSI \\
\hline Hair removal & $\begin{array}{l}\text { Removal does not reduce infection - if necessary } \\
\text { clippers reduce SSI incidence compared to shaving }\end{array}$ \\
\hline Patient preoperative showering: & $\begin{array}{l}\text { Known reduction in skin bioburden but inconclusive } \\
\text { evidence of reduction in SSI }\end{array}$ \\
\hline Antibiotics & $\begin{array}{l}\text { Prophylactic antibiotics should be given for implant } \\
\text { surgery during anaesthetic induction (earlier if } \\
\text { tourniquet to be used-post op regimes contentious }\end{array}$ \\
\hline \multicolumn{2}{|l|}{ Inter operative } \\
\hline Skin prep in theatre: & $\begin{array}{l}\text { Current evidence inconclusive for any single best agent } \\
\text { - alcohol likely to play an important role in efficacy }\end{array}$ \\
\hline Adhesive occlusive drapes & $\begin{array}{l}\text { lodine impregnated drapes may reduce SSI - non } \\
\text { impregnated drapes may actually increase SSI incidence }\end{array}$ \\
\hline Diathermy skin incision & No evidence of reduced SSI compared to scalpel incision \\
\hline Procedure length & $\begin{array}{l}\text { 个 duration of procedure is directly linked to } \uparrow \text { risk of } \\
\text { SSI }\end{array}$ \\
\hline Wound closure technique & $\begin{array}{l}\text { Subcuticular suture allows greatest perfusion of skin } \\
\text { edges but no evidence of reduced SSI }\end{array}$ \\
\hline Antimicrobial-coated sutures & $\begin{array}{l}\text { Triclosan-coated sutures appear to reduce incidence of } \\
\text { SSI compared to conventional sutures }\end{array}$ \\
\hline Theatre discipline & $\begin{array}{l}\text { Staff numbers, number of door openings, noise levels in } \\
\text { theatre all linked to increased SSI }\end{array}$ \\
\hline Oxygen tension & $\begin{array}{l}\uparrow \text { concentrations of inspired oxygen result in } \downarrow \text { SSI } \\
\text { rates }\end{array}$ \\
\hline Hypothermia & $\begin{array}{l}\uparrow \mathrm{O}_{2} \text { demand and acidosis causing poor perfusion of } \\
\text { end organs }\end{array}$ \\
\hline
\end{tabular}

\section{Clinical Presentation}


An SSI may present at any time up to a year following prosthetic implant surgery, infections of implants after this time are considered to be due to a cause not related to the implant procedure (eg Haematogenous spread). Early prosthetic infections occurring within 30 days of a surgical procedure, frequently present with pain, fever, leucocytosis, bacteraemia and cellulitis. In some instances there may be abscess formation or a sinus tract discharging pus. Intermediate infections may occur between one and three months following surgery with a similar presentation to early infections. Late infections present more than three months post- operatively. They are often indolent in nature and are frequently caused by less virulent bacterial strains often presenting with a continually discharging sinus but no clinical signs of sepsis and often negative cultures.

\section{Orthopaedic}

Orthopaedic prosthetic infections may have a delayed presentation when less virulent organisms are responsible and diagnoses may be more problematic. Symptoms such as persistent joint pain, deterioration in function and occasionally loosening of the prosthesis may be present. Differentiating such chronic infections from aseptic failure or loosening is difficult.

\section{Vascular}

The presentation of vascular graft infections depends on the anatomical position of the graft and endovascular procedures have the same incidence of prosthetic infection as open implant procedures. Vascular grafts infections may present with chronic or acute pain and may be complicated by fistula or pseudo-aneurysm formation, bleeding at the anastomotic site, thrombosis of the graft or peripheral embolisation. Occasionally osteomyelitis or nonhealing ulcers may develop in tissues distal to the grafts.

\section{Cardiac}

Infections related to cardiac implantable devices can be divided into two categories: pocket infections (infection in the subcutaneous device pocket or the subcutaneous leads) and deeper infections (involving the trans-venous portions of the leads and/ or endovascular infection such as endocarditis). Deeper infections often give rise to late presentations and may feature a persistent or relapsing bacteraemia without obvious source or occasionally distal or pulmonary emboli.

\section{Diagnosis}

Timely diagnosis of prosthetic implant infections is crucial in preventing morbidity and mortality. Accuracy of diagnosis is particularly important in avoiding significant insult from the implant removal and or revision surgery that such infections necessitate. The presence of a surgical prosthesis should provoke a high level of suspicion in the clinician assessing any patient with ongoing symptoms relating to surgical sites or infective symptoms of unknown origin. Initial assessment should include blood sampling for inflammatory markers though a leucocytosis and elevated CRP would be expected in the immediate post-operative period, and serial measures to detect a trend are more helpful than solitary results. 
Microbiological culture specimens must be sent from all relevant sites for gram stain and culture. Prior antibiotic administration may result in a failure to demonstrate a pathogen in culture specimens making it imperative that, where possible, cultures should be obtained before commencing treatment. Blood cultures should be obtained from several sites especially if haematogenous spread is a possibility.

Surface swabs from skin around incision sites are often difficult to interpret due to colonising organisms acting as sample contaminants. Fine needle aspiration of surgical wound cellulitis has been advocated as more likely to identify a single causative organism when compared to surface skin swabs [12] though this is not common practice in most units to date. Cultures of aspirated peri-prosthetic fluid collection or synovial fluid remain the most reliable methods of diagnosis. Peri-prosthetic tissue biopsies may also be considered in some cases.

In cases where drained collections reveal white cells but no pathogens, S. epidermidis should be considered.

\section{Diagnostic Imaging}

The use of imaging for the diagnosis of prosthetic infections must be tailored to the patient and the prosthesis type as each modality has its sensitivities and specificities as well as advantages and disadvantages.

X-rays have little role in the diagnosis of prosthetic infections with the exception of orthopaedic implants (Fig 1). Joint loosening may be diagnosed on plain radiography but serial examinations may be required. For more accurate examination of the interface between the cement and bone, MRI may be necessary.

\section{Ultrasound (US)}

US is radiation free but is highly operator dependant and whilst useful in detecting complications due to infection (pseudo-aneurysm, peri-prosthetic collection) has a limited role in detecting low grade infection of prostheses. US echocardiogram may assist in the diagnosis of infective endocarditis of a prosthetic valve by the identification of vegetations, thrombus, or a pericardial effusion. Sensitivity for vegetations is higher with transoesophageal (85-90\%) rather than trans-thoracic ( 75\%) echocardiogram[13].

\section{Computed tomography and Magnetic resonance scanning}

Both are less operator dependant that USS. Scans can be obtained quickly and large areas imaged accurately (Fig 2). It has been reported that MRI and CT have comparable $95 \%$ specificity and $55 \%$ sensitivity for prosthetic vascular graft infection. The low sensitivity in both cases is due to difficulties differentiating between the normal post operative changes of haematoma, but the presence of these signs should be considered abnormal after 6-8 weeks[14].

The use of CT is limited by stainless steel alloy devices which can result in image artefacts which may compromise the value of the scan though artefact is less of a problem with the 
newer titanium and nitinol implants and stents / grafts. CT does result in a very high radiation dose and the requirement for nephrotoxic contrast is relatively contraindicated in chronic kidney disease. MRI can only be performed in patients with non-metallic implants or those safe for MRI such as titanium or tantalum. The presence of metallic implants or prostheses results in significant image artefacts which may render the results uninterpretable. Equally MRI is contraindicated in patients with cardiac devices such as pacemakers.

\section{PET scanning}

Positron emission tomography-computed tomography with ${ }^{18}$ F-fludeoxyglucose (FDG-PETCT) has been widely used in detection of cancer metastatic deposits by highlighting areas of enhanced glucose metabolism. Recent advances have suggested this technique to be highly sensitive in the detection and exclusion of peri-prosthetic infections in many areas. Very promising results for specificity and sensitivity have been reported for aortic and peripheral vascular graft infections [15] peri-prosthetic infection in joint replacement surgery[16] and prosthetic cardiac valves[17].

\section{Leucocyte scintigraphty and SPECT-CT}

Tc99m-labelled white cell scans (Fig 3), may prove valuable in cases where there is difficulty establishing whether or not the prosthesis is infected. However, despite the very high sensitivity (around 100\%) for detection of infection, within the first year after surgery there is a low specificity (75\%) due to remodelling around the prosthesis.

Further limitations include the production of planar, or two-dimensional images with no spatial resolution and limited landmarks for localization .

SPECT-CT combines the use of radioneucleotide tracers with the 3D spatial resolution of CT scanning and can address problems due to radiotracers with extensive distribution that produce an unfavourable signal "noise" making discrimination of pathology from normal activity difficult[18]. The use of SPECT in cancer is well established and it will probably be increasingly employed in the investigation of infections in the future a greater experience becomes available. Data in prosthetic infection to date is limited to case studies and short series.

\section{Management}

Following initial diagnosis the management of prosthetic infections should comprises a combination of two or more antibiotics providing a broad activity spectrum at lower concentrations to improve efficacy. Treatment should ideally then be rationalised and targeted if and when culture and sensitivities confirm the pathogen and preferred antibiotic agent. Unfortunately the prevalence of multi-resistant strains of Coagulase negative staphylococci has increased dramatically over that past couple of decades. As with MRSA $\beta$ lactam resistance is the most common. This increase in resistance has emphasised the need for the development of new antibiotics such as linezolid and tigecycline. Antibiotic treatment therefore typically has little or no effect against the biofilm on colonised prostheses. 
Management of patients with prosthetic infections often necessitates more aggressive surgical treatment. Debridement is often the first step and involves the removal of haematoma, devitalised tissue, scar tissue and sinus tracts. Multiple debridements or explantation of the infected prosthesis and revision may be necessary. In some situations debridement and or explantation is not always feasible given patient co-morbidities and surgical risk in which case palliation and long term antibiotic therapy may be most appropriate.

\section{Orthopaedic}

Success rates with debribement are reported to be in the region of $60-80 \%$ in selected patients with prosthetic arthroplasty prostheses[19]. Explantation and revision of an infected arthroplasty is the subject of much debate. Many surgeons still favour a 2 stage revision involving the use of a temporary antibiotic cement spacer. However comparable infection eradication has been reported in a one stage direct exchange procedure, predominantly using antibiotic loaded cement. Further studies have shown that antibiotic bone compound has better storage capacities and pharmacological kinetics than loaded cement and may reduce the re-infection risk. In a limited number of cases where these measures fail, arthrodesis or even amputation may be required.

\section{Vascular}

The morbidity and mortality of infected vascular grafts is dependent on the location of the graft. The literature reports mortality rates ranging from $6-75 \%$ and an amputation rate of 21- 70\% [20]. Despite high mortality rates associated with resection of infected grafts (25$35 \%$ in aortic grafts) the patient outcomes are thought to be better following surgical as opposed to conservative management. It is therefore clear that management decisions for patients with major arterial grafts are problematic. Circumstances may mandate that surgical revision/ excision is carried out, for example in patients with false aneurysm formation or bleeding at the anastomosis, or patients who have developed arteriovenous or enteric fistulae. If removal of a vascular graft is deemed imperative, due to infection, then the surgical options are complex and include in situ graft replacement- using autogenous, prosthetic or impregnated grafts, or removal with extra-anatomic bypass.

\section{Cardiac}

In cases of pocket infection associated with intra-cardiac devices it may be possible to treat with antibiotics and simply drain any superficial collections, however leads have often already become colonised and therefore antibiotic treatment alone is rarely effective in preventing recurrence. For patients who are deemed fit the device can be removed and rhythm temporarily controlled using transvenous pacing wires or an external pacing vest. The new device is then placed on the contralateral side between 2 and 6 weeks later. Patients deemed to be unfit for such a procedure may need intermittent antibiotic therapy for the duration of their life.

Infections of prosthetic heart valves have a mortality rate of around $30 \%$. Surgical replacement of the valves can be performed only in specialist centres and is often only carried out if medical therapy has failed or is the patient is displaying cardiovascular or cerebrovascular complications. 


\section{Current research and developments}

New prostheses are being developed to employ the same principles as antimicrobial coated sutures. Neurosurgical shunts impregnated with antibiotic agents have been shown to be clinically and cost effective in reducing infective morbidity and mortality[21]. In orthopaedic practice antimicrobial or antibiotic coated implants and antibiotic bonded cement have shown promising results in combination with systemic antibiotics, in animal models and early human trials in prevention of implant associated infections[22, 23]. Early trials in antimicrobial coating of hernia mesh implants have been reported [24] as well as coating of vascular grafts with Triclosan and Rifampicin resulting in significantly reduced microbial load and rate of surgical site infections in animal models[25].

Concerns have been raised that antibiotic bonding may lead to an increased level of pathogen resistance and work is also underway to create new biological prostheses, which provoke no foreign body reaction thus negating the need for prophylactic antimicrobial impregnation or coatings. In vascular surgery biosynthetic devices composed of ovine collagen grown around a polyester mesh template have shown promising medium term results for revision implant surgery with implantation into infected surgical fields[26]. Early human studies are also underway using grafts grown in-vitro from banked human derived vascular smooth muscle cells which are then de-cellularised to eliminate risk of host rejection following implantation. Results of phase 1 trials are awaited.

Biological meshes have been widely used for several years particularly for repair of abdominal wall defects. These are comprised of acellular extracts obtained from either human or non-human, usually porcine, sources. Significantly higher costs means their use is typically reserved for use in contaminated or dirty wounds though this use is unlicensed and there is a paucity of evidence to support this use[27].

\section{Conclusion}

Prosthetic implant infections result in significant morbidity and mortality for patients and huge costs to healthcare systems. Due to the serious implications it is imperative that all appropriate preventative measures against SSI and implant infection are employed. Surgeons must work closely with other healthcare professionals to optimise patient, surgical, anaesthetic and environmental risk factors. Newer antimicrobial or biological implants may also help to reduce the incidence of this devastating complication.

1. Smyth, E.T., et al., Four country healthcare associated infection prevalence survey 2006: overview of the results. J Hosp Infect, 2008. 69(3): p. 230-48.

2. Leaper, D. and K. Ousey, Evidence update on prevention of surgical site infection. Curr Opin Infect Dis, 2015. 28(2): p. 158-63.

3. Moxey, P.W., et al., Lower extremity amputations--a review of global variability in incidence. Diabet Med, 2011. 28(10): p. 1144-53.

4. Seeger, J.M., Management of patients with prosthetic vascular graft infection. Am Surg, 2000. 66(2): p. 166-77. 
5. Del Pozo, J.L. and R. Patel, Clinical practice. Infection associated with prosthetic joints. N Engl J Med, 2009. 361(8): p. 787-94.

6. Margey, R., et al., Contemporary management of and outcomes from cardiac device related infections. Europace, 2010. 12(1): p. 64-70.

7. Hinchliffe, R.J. and W.J. Jeffcoate, Diabetes: Diabetes mellitus: are amputation rates falling in England? Nat Rev Endocrinol, 2010. 6(8): p. 422-4.

8. Moxey, P.W., et al., Epidemiological study of lower limb amputation in England between 2003 and 2008. Br J Surg, 2010. 97(9): p. 1348-53.

9. Berendt, A.R., et al., Specific guidelines for treatment of diabetic foot osteomyelitis. Diabetes Metab Res Rev, 2008. 24 Suppl 1: p. S190-1.

10. Hinchliffe, R.J., et al., Specific guidelines on wound and wound-bed management. Diabetes Metab Res Rev, 2008. 24 Suppl 1: p. S188-9.

11. de Lusignan, S., F. Hogg, and R.J. Hinchliffe, Getting the signal to noise ratio right in the management of diabetes in primary care: time to stratify risk and focus on outcomes rather than process. Inform Prim Care, 2010. 18(4): p. 219-21.

12. Parikh, A.R., et al., Diagnostic Fine-Needle Aspiration in Postoperative Wound Infections is More Accurate at Predicting Causative Organisms than Wound Swabs. Annals of The Royal College of Surgeons of England, 2007. 89(2): p. 166-167.

13. Hinchliffe, R.J., et al., The effect of haemodialysis on transcutaneous oxygen tension in patients with diabetes-a pilot study. Nephrol Dial Transplant, 2006. 21(7): p. 19813.

14. Eikelboom, J.W. and G.J. Hankey, Low molecular weight heparins and heparinoids. Med J Aust, 2002. 177(7): p. 379-83.

15. Sah, B.R., et al., Diagnostic Performance of F-FDG-PET/CT in Vascular Graft Infections. Eur J Vasc Endovasc Surg, 2015.

16. Chryssikos, T., et al., FDG-PET Imaging Can Diagnose Periprosthetic Infection of the Hip. Clinical Orthopaedics and Related Research, 2008. 466(6): p. 1338-1342.

17. Stevens, P.E., et al., Chronic kidney disease management in the United Kingdom: NEOERICA project results. Kidney Int, 2007. 72(1): p. 92-9.

18. Feldman, H.I., et al., Predictors of successful arteriovenous fistula maturation. Am J Kidney Dis, 2003. 42(5): p. 1000-12.

19. Kuiper, J.W.P., et al., Treatment of acute periprosthetic infections with prosthesis retention: Review of current concepts. World Journal of Orthopedics, 2014. 5(5): p. 667-676.

20. Erb, S., et al., Surgical and antimicrobial treatment of prosthetic vascular graft infections at different surgical sites: a retrospective study of treatment outcomes. PLoS One, 2014. 9(11): p. e112947.

21. Edwards, N.C., et al., Cost-consequence analysis of antibiotic-impregnated shunts and external ventricular drains in hydrocephalus. J Neurosurg, 2015. 122(1): p. 13947.

22. Folsch, C., et al., Coating with a novel gentamicinpalmitate formulation prevents implant-associated osteomyelitis induced by methicillin-susceptible Staphylococcus aureus in a rat model. Int Orthop, 2014.

23. Shirai, T., et al., Antimicrobial megaprostheses supported with iodine. J Biomater Appl, 2014. 29(4): p. 617-23.

24. Suarez-Grau, J.M., et al., Antibiotic embedded absorbable prosthesis for prevention of surgical mesh infection: experimental study in rats. Hernia, 2014. 
25. Hernandez-Richter, T., et al., Rifampin and Triclosan but not silver is effective in preventing bacterial infection of vascular dacron graft material. Eur J Vasc Endovasc Surg, 2003. 26(5): p. 550-7.

26. Wiltberger, G., et al., Mid-and long-term results after replacement of infected peripheral vascular prosthetic grafts with biosynthetic collagen prosthesis. J Cardiovasc Surg (Torino), 2014. 55(5): p. 693-8.

27. Cross, W., A. Kumar, and G. Chandru Kowdley, Biological mesh in contaminated fields--overuse without data: a systematic review of their use in abdominal wall reconstruction. Am Surg, 2014. 80(1): p. 3-8. 